\title{
Plasma glutathione as a risk marker for the severity and functional outcome of acute atherothrombotic and cardioembolic stroke
}

\author{
Alexander Vladimirovich Ivanov ${ }^{{ }^{*}} \mathbb{D}$, Marina Yurievna Maksimova², Ksenya Alexandrovna Nikiforova', \\ Fatima Ramazanovna Ochtova ${ }^{3}$, Ekaterina Taymurazovna Suanova ${ }^{3}$, Valery Vasil'evich Alexandrin', \\ Maria Petrovna Kruglova ${ }^{4}$, Mikhail Aleksanrovich Piradov ${ }^{2}$ and Aslan Amirkhanovich Kubatiev ${ }^{1,5}$
}

\begin{abstract}
Objective: Glutathione (GSH) is a major intracellular thiol-containing antioxidant. We tried to determine whether blood plasma GSH level is a marker for the severity of the two subtypes of acute stroke (large-artery atherosclerosis, LA and cardioembolic, CE). Forty-three patients with LA and 36 patients with CE aged 65 (47-82) years were included in the study. Thirty-one patients with cerebral microangiopathy were included for comparison. Total ( $\mathrm{t}$ ) and reduced (r) GSH levels were determined at admission. Neurological deficit was assessed by the National Institutes of Health Stroke Scale (NIHSS) on the first day, functional outcome and independence were assessed by the modified Rankin scale (mRs) and Bartel index (BI), respectively, after 21 days.

Results: The tGSH and rGSH levels in acute stroke were significantly lower than cerebral microangiopathy patients. Low tGSH $(\leq 1.45 \mu \mathrm{M})$ and $\mathrm{rGSH}(\leq 30 \mathrm{nM})$ levels were risk markers for stroke severity at admission (NIHSS $>10)$ in patients with LA: age and gender adjusted odds ratio (AOR) was 4.95, 95\% coincidence interval (CI) 1.31-18.7, $\mathrm{AOR}=9.141, \mathrm{Cl} 1.84-45.3$ for $\mathrm{t}$ - and $\mathrm{rGSH}$, respectively. A low level of rGSH $(\leq 30 \mathrm{nM})$ was found as risk marker for functional independence $(\mathrm{BI} \leq 60$ : $\mathrm{AOR}=15.9, \mathrm{Cl} 2.22-114.2)$ in patients with LA. Low tGSH level $(\leq 1.1 \mu \mathrm{M})$ was associated with the reduction of poor outcome risk ( $m R s>2$ : $A O R=0.154, C l 0.029-0.809$ ) in CE group.
\end{abstract}

Conclusions: Low t- and rGSH levels may be considered potential risk markers for severity and insufficient functional independence in LA. Conversely, low tGSH level reduce the risk of poor stroke outcome only for CE.

Keywords: Cardioembolism, Glutathione, Ischemic stroke, Large-artery atherosclerosis, Biomarkers

\section{Background}

Ischemic stroke (IS) remains one of the most significant problems of healthcare; therefore, an important task is to search for markers and factors associated with its severity and prognosis. Atherothrombotic (large-artery atherosclerosis, LA) and cardioembolism (CE) subtypes account for almost half of the cases of ischemic stroke

\footnotetext{
*Correspondence: ivanov_av82@mail.ru

${ }^{1}$ Department of Molecular and Cellular Pathophysiology, Institute of General Pathology and Pathophysiology, Baltiyskaya st., 8, 125315 Moscow, Russia

Full list of author information is available at the end of the article
}

in the European population [1]. Oxidative stress plays an important role in the pathogenesis of both ischemic brain damage and the response to it [2, 3]. Majority of antioxidants are reduced immediately after an acute ischemic stroke [4]. Diagnostic and prognostic capabilities of various indicators of oxidative stress and antioxidant defense potential (total antioxidant capacity, F2-isoprostanes, oxygen radical absorbance capacity, glutathione peroxidase, malonedialdehyde, advanced glycation end products, and protein oxidation products etc.) in stroke are being intensively studied [5-8]. Glutathione ( $\gamma$-glutamylcysteinyl-glycine tripeptide, GSH) is the main low 
molecular weight aminothiol in cells, participating in a variety of vital processes, including protecting the thiol groups of proteins from oxidative damage and neutralizing reactive oxygen species. Glutathione peroxidasesensitive reactive oxygen species play an important role in regulation of cell death during cerebral ischemia-reperfusion by modulating intrinsic neuronal sensitivity, as well as brain inflammatory reactions [9]. In the brain, it is predominantly in a reduced form in high concentration ( 1-3 mM, [10]), as a result of which GSH may be a physiologic reservoir of glutamate neurotransmitter [11]. At the same time, its level in blood plasma is much lower (approximately $3 \mu \mathrm{M}$ [12]) and is represented mainly by forms oxidized to disulfides [13].

The question of the possible association of the GSH level in blood plasma with the risk and prognosis of ischemic stroke of various subtypes is still very poorly studied, despite the important role of this metabolite. A decrease in reduced and an increase in oxidized GSH was shown in the brain on rat models of temporary global and focal cerebral ischemia $[14,15]$. In patients with acute stroke, in general, there was no significant change in relation to the GSH content in the stroke region compared with the corresponding measurement from the contralateral hemisphere [16]. However, in the blood plasma, an increase in GSH levels was previously found in patients with acute stroke on the first day; however, no statistically significant correlation between GSH levels and Canadian Neurological Scale (CNS) scores was detected $[17,18]$. It has recently been shown that the reduced GSH concentration in stimulated whole saliva decreased with a high degree of confidence $(p<0.0001)$ at subacute stroke phase [19]. In the present work, we studied the effect of the total and reduced forms of GSH (tGSH and rGSH correspondingly) in blood plasma on the risk of increased neurological deficit and unfavorable functional outcome in patients with primary acute LA and CE stroke.

\section{Methods \\ Patients}

The study included 79 patients with primary IS in the carotid artery basin who were admitted in the first 10-24 h after the development of neurological disorder. Thirty-one patients with cerebral microangiopathy were included in the comparison group. Neither the patients nor the controls had a previous cerebrovascular event (IS, cerebral hemorrhage, or transient ischemic attack). Patients were admitted to the neurology department of the Research Center of Neurology. All procedures performed in the study involving human participants were in accordance with the ethical standards of our institutional research committee and with the 1964 Helsinki declaration and its later amendments or similar ethical standards. This study was approved by the local ethics committee of the Scientific Center of Neurology and was conducted with the informed consent of patients or their first-line relatives.

The diagnosis was based on clinical findings, neurologic examination, magnetic resonance imaging (MRI) of the brain, color duplex examination of cerebral arteries, and transthoracic echocardiography. The diagnosis of cerebral microangiopathy was based on anamnestic data on the long course of mild and moderate non crisis arterial hypertension over 5-year duration period, hypertensive cerebral crises, impaired cognitive functions (cognitive impairment), MRI data: silent small deep brain infarctions in the subcortical structures, and the silent small deep infarcts in the microstructural damage of white mater.

All stroke patients underwent magnetic resonance (MR) imaging of the brain using the Magnetom Verio (Siemens) and Magnetom Symphony (Siemens) devices with magnetic induction values of $3,1.5$, and 1.5 Tesla, respectively. MR angiography was performed in a 3D-TOF mode to detect the intracranial artery pathology. Brain infarction was defined as a focus of increased MR signal intensity in the T2, T2 d-f, and DWI modes with a reduced diffusion coefficient on the ADC map.

The stroke subtype has been classified according to the TOAST (Trial of ORG 10,172 in Acute Stroke Treatment) classification [20]. The main criteria for inclusion in the study were: acute LA and CE; hospitalization within the first $24 \mathrm{~h}$ from the onset of a neurological symptom; and age 45-85 years. The exclusion criteria were: a delay of more than $24 \mathrm{~h}$ between onset of symptoms and diagnosis of ischemic stroke; lacunar infarct; undetermined causes of stroke; hemorrhagic stroke; epilepsy; pregnancy; type 1 diabetes; acute myocardial infarction; permanent cardiac pacing; decompensated renal, hepatic, or respiratory failure; congestive heart failure of a III-IV functional class; oncological diseases; chronic inflammatory, autoimmune, and hematological disorders; and infectious diseases within 15 days after a stroke. Neurologic impairment severity was evaluated using National Institutes of Health Stroke Scale (NIHSS) on the first day [21], and evaluation of functional recovery was performed using modified Rankin scale (mRs) on the 21st day of ischemic stroke [22]. An mRs score of $>2$ was used to define a poor functional outcome. The patients' activities of daily living were evaluated using the Barthel Index (BI) [23] on the 21st day. A BI score of $\leq 60$ was consistent with a decline in functional independence. The patients underwent basic treatment with antihypertensive drugs, antiplatelet agents, anticoagulants, statins. 


\section{Aminothiols determination}

Venous blood was collected on admission into tubes containing sodium citrate $(0.38 \%)$ and centrifuged at $3000 \times g$ for $3 \mathrm{~min}$. The plasma samples were collected, frozen at $-80{ }^{\circ} \mathrm{C}$, and stored until analysis. Determination of the total and reduced forms of blood plasma aminothiols (GSH, cysteine, and homocysteine) was carried out by liquid chromatography (Acquity UPLC $\mathrm{H}$-class system, Waters, Milford, Massachusetts, USA) as described previously [13].

\section{Statistical analysis}

Data collection and primary processing (identification and integration of the chromatographic peaks) were performed using MassLynx v4.1 (Waters, USA). Statistical data analysis was performed using SPSS Statistics v. 22 (IBM, USA). Intergroup comparisons were performed using the ANOVA Tamhane's T2 and binomial tests, the association of indicators with each other was identified using the Spearman's rank correlation coefficient $(\rho)$. To identify risk relative risk (RR), unadjusted odds ratio (OR), age-, and gender-adjusted odds ratio (AOR) were calculated. Differences were considered significant at a significance level of $p<0.05$.

\section{Results}

A total of 79 IS and 31 cerebral microangiopathy patients were examined, and their general characteristics have been shown in Table 1. IS patients showed significant reductions in tGSH and, exceptionally, rGSH levels. In addition, CE patients had lower total cysteine and homocysteine levels than LA. Significant differences were found between groups CE and LA with regards to age, sex, and the incidence of chronic cerebral ischemia.

The levels of tGSH and rGSH were associated with each other, both in the LA group $(\rho=0.412, p=0.007)$ and in the CE group $(\rho=0.55, p=0.001)$. In the CE group, there was a significant positive association of tGSH with total cysteine $(\rho=0.49, p=0.002)$ and homocysteine $(\rho=0.472, p=0.004)$, which was absent in the LA group. There were no significant differences in rGSH levels among LA and CE.

On dividing both groups into subgroups with low $(\leq 1.45 \mu \mathrm{M})$ and high $(>1.45 \mu \mathrm{M})$ levels of $\mathrm{tGSH}$, it was found that an increased risk of stroke severity (NIHSS > 10) is characteristic only of patients with LA with low tGSH, and in the case of CE this is not observed in unadjusted and adjusted models (Table 2). With a decrease in the tGSH threshold to $1.1 \mu \mathrm{M}$, which corresponds to the median of its level in the group of patients with $\mathrm{CE}$, again, there was no increase in this risk in $\mathrm{CE}$ patients (data not shown).

Table 1 General characteristics of patients

\begin{tabular}{|c|c|c|c|c|}
\hline Variables & Cerebral microangiopathy & LA & CE & $p^{* *}$ \\
\hline N & 31 & 43 & 36 & \\
\hline Age, years & $69[60 ; 75]$ & $62[55 ; 66]^{\#}$ & $68[60 ; 75]$ & 0.01 \\
\hline Sex, $\%$ man & 39 & $79^{*}$ & 39 & 0.00014 \\
\hline Chronic cerebral ischemia & 31 [100\%] & $11[25.6 \%]^{*}$ & $18[50.0 \%]^{*}$ & 0.025 \\
\hline NIHSS on admission & - & $9[5 ; 12]$ & $12[7 ; 13]$ & \\
\hline $\mathrm{NIHSS}>10, n$ & - & $19(44.2 \%)$ & $19(52.8 \%)$ & \\
\hline mRs (21st day) & - & $3[1 ; 4]$ & $3[2 ; 4]$ & \\
\hline $\mathrm{mRs}>2, n$ & - & $24(55.8 \%)$ & $21(58.3 \%)$ & \\
\hline BI (21st day) & - & $60[45 ; 86]$ & $75[30 ; 85]$ & \\
\hline $\mathrm{Bl}<61, n$ & - & $22(51.1 \%)$ & $19(52.8 \%)$ & \\
\hline $\mathrm{tGSH}, \mu \mathrm{M}$ & $3.1[2.1 ; 3.7]$ & $1.6[1.1 ; 2.7]^{\#}$ & $1.1[0.8 ; 2.2]^{\# \#}$ & 0.00001 \\
\hline total cysteine, $\mu \mathrm{M}$ & $267[230 ; 322]$ & $325[287 ; 363]$ & $172[135 ; 336]^{\& \&}$ & 0.00009 \\
\hline total homocysteine, $\mu \mathrm{M}$ & $13.0[11.4 ; 15.9]$ & $14.4[12.2 ; 19.8]$ & $8.7[5.3 ; 14.1]^{\&}$ & 0.0017 \\
\hline $\mathrm{rGSH}, \mathrm{nM}$ & $240[180 ; 370]$ & $42[25 ; 68]^{\# \#}$ & $39[25 ; 57]^{\# \#}$ & $<0.00001$ \\
\hline
\end{tabular}

All values represented as median [Q1; Q3]

$B$ I Bartel index, $m$ Rs modified Rankin scale, NIHSS National Institutes of Health Stroke Scale, $r G S H$ reduced glutathione, $t G S H$ total glutathione

${ }^{*} p<0.05$ (binomial test)

**Tamhane test for all groups

\# Tamhane test $p<0.05, " \#<0.005$ compared to cerebral microangiopathy group

\& Tamhane test $p<0.05,{ }^{\& \&} p<0.001$ compared to LA group 
Similar results were obtained with a division of patients by the level of rGSH. On dividing patients according to the $30 \mathrm{nM}$ rGSH threshold level, it was found that the risk of stroke severity increased in patients with low rGSH levels in group LA, but not in group CE (Table 3 ).

Unadjusted model analysis also showed that low tGSH level $(\leq 1.1 \mu \mathrm{M})$ at admission was associated with a risk of 3-week poor outcome $(\mathrm{mRs}>2)$ for LA stroke (Table 4$)$. However, taking into account gender and age, these results turned out to be insufficiently reliable, despite the rather high value of AOR. In contrast, reduced risk of poor outcome was observed in patients with low tGSH levels in CE group on adjusted model (Table 4). In turn, a rather pronounced association of low rGSH levels $(\leq 30 \mathrm{nM})$ with insufficient functional state of patients was found in group LA, but not in group CE (Table 4).
Due to the fact that patients in the CE group were generally older than patients in the LA group, we analyzed the association of $\mathrm{t}$ - and rGSH levels with age. In the whole cohort of patients, the level of tGSH had a slight negative association with age $(\rho=-0.237, p=0.035)$, but it was not found in the LA group ( $\rho=-0.061, p=0.698)$, and in the CE group it was not significant $(\rho=-0.201$, $p=0.239$ ). Morever, there was no significant association between rGSH level and age in the LA group ( $\rho=-0.112, p=0.481)$, and in the CE group it was negative ( $\rho=-0.357, p=0.038$ ). Thus, we have no reason to believe that age may be a significant factor affecting GSH levels in patients with LA.

We also conducted a comparative analysis of $\mathrm{t}$ - and rGSH levels among women and men, because the latter significantly prevailed in the group with LA. In total, 11 of 48 men had a $\mathrm{tGSH} \leq 1.1 \mu \mathrm{M}$, while of 31 women there

Table 2 Impact of tGSH on the risk of increased neurologic deficits (NIHSS) on admission in stroke patients

\begin{tabular}{|c|c|c|c|c|c|c|c|c|}
\hline \multirow[t]{2}{*}{ tGSH, $\mu \mathrm{M}$} & \multicolumn{4}{|l|}{ LA } & \multicolumn{4}{|l|}{ CE } \\
\hline & NIHSS $<11$ & NIHSS $>10$ & OR (Cl 95\%) & AOR (Cl 95\%) & NIHSS $<11$ & NIHSS $>10$ & OR (Cl 95\%) & AOR (CI 95\%) \\
\hline$\leq 1.45$ & 6 & 12 & $5.14(1.38-19.1)$ & $4.952(1.31-18.7)$ & 8 & 13 & $1.42(0.568-2.64)$ & $1.166(0.281-4.83)$ \\
\hline$>1.45$ & 18 & 7 & & & 7 & 8 & & \\
\hline
\end{tabular}

AOR adjusted odds ratio, CE cardioembolic stroke, CI 95\% 95\% coincidence interval, LA large-artery atherosclerosis, NIHSS National Institutes of Health Stroke Scale, OR odds ratio, $t G S H$ total glutathione

Table 3 Impact of rGSH on the risk of increased neurologic deficits on admission in patients with acute stroke

\begin{tabular}{|c|c|c|c|c|c|c|c|c|}
\hline \multirow[t]{2}{*}{$\mathrm{rGSH}, \mathrm{nM}$} & \multicolumn{4}{|l|}{ LA } & \multicolumn{4}{|l|}{ CE } \\
\hline & NIHSS $<11$ & NIHSS > 10 & OR (Cl 95\%) & AOR (Cl 95\%) & NIHSS $<11$ & NIHSS $>10$ & OR (Cl 95\%) & AOR (Cl 95\%) \\
\hline$\leq 30$ & 3 & 9 & $6.3(1.39-28.5)$ & $9.141(1.84-45.3)$ & 3 & 8 & $3.39(0.725-15.9)$ & $4.64(0.783-27.5)$ \\
\hline$>30$ & 21 & 10 & & & 14 & 11 & & \\
\hline
\end{tabular}

AOR adjusted odds ratio, CE cardioembolic stroke, CI 95\% 95\% coincidence interval, LA large-artery atherosclerosis, NIHSS National Institutes of Health Stroke Scale, OR odds ratio, $r G S H$ reduced glutathione

Table 4 Influence of t- and rGSH levels on the risk of poor functional outcome and dependence in daily care in stroke patients (21 days after stroke)

\begin{tabular}{|c|c|c|c|c|c|c|c|c|}
\hline \multirow[t]{2}{*}{ GSH } & \multicolumn{4}{|l|}{ LA } & \multicolumn{4}{|l|}{ CE } \\
\hline & $\mathrm{mRs}<3$ & $\mathrm{mRs}>2$ & OR (Cl 95\%) & AOR (CI 95\%) & $\mathrm{mRs}<3$ & $\mathrm{mRs}>2$ & OR (Cl 95\%) & AOR (Cl 95\%) \\
\hline$\leq 1.1^{\mathrm{a}}$ & 1 & 8 & $9.0(1.01-80.0)$ & $9.288(0.859-100.4)$ & 10 & 7 & $0.25(0.061-1.02)$ & $0.154(0.029-0.809)$ \\
\hline$>1.1^{\mathrm{a}}$ & 18 & 16 & & & 5 & 14 & & \\
\hline \multirow[t]{2}{*}{ GSH } & \multicolumn{4}{|l|}{ LA } & \multicolumn{4}{|l|}{ CE } \\
\hline & $\mathrm{BI}<61$ & $\mathrm{BI}>60$ & OR (Cl 95\%) & AOR (CI 95\%) & $\mathrm{BI}<61$ & $\mathrm{BI}>60$ & OR (Cl 95\%) & AOR (Cl 95\%) \\
\hline$\leq 30^{b}$ & 2 & 10 & $8.64(1.59-46.8)$ & $15.9(2.22-114.2)$ & 6 & 6 & $0.692(0.168-2.85)$ & $0.602(0.132-2.73)$ \\
\hline$>30^{b}$ & 19 & 11 & & & 9 & 13 & & \\
\hline
\end{tabular}

$A O R$ adjusted odds ratio, BI Bartel index, CE cardioembolic stroke, CI 95\% 95\% coincidence interval, GSH glutathione, LA large-artery atherosclerosis, $m R s$ modified Rankin scale, NIHSS National Institutes of Health Stroke Scale, OR odds ratio

a Total, $\mu \mathrm{M}$

${ }^{\mathrm{b}}$ Reduced, $\mathrm{nM}$ 
were 12 who had the same tGSH $(\mathrm{RR}=0.59, p=0.131)$. Of 47 men, 15 had $\mathrm{rGSH} \leq 30 \mathrm{nM}$, while of 29 women there were $8(R R=1.16, p=0.689)$. These results show that there was no significant differences in the incidence of low levels of $\mathrm{t}$ - and rGSH between women and men.

As in the LA group, the incidence of chronic cerebral ischemia was significantly lower than in the CE group; we also investigated the effect of this factor on the incidence of low levels of $\mathrm{t}$ - and rGSH in a cohort of patients. There was no significant association between the presence of chronic ischemia and low levels $(\leq 1.45 \mu \mathrm{M})$ of tGSH $(\mathrm{RR}=1.43, p=0.28)$ and $\mathrm{rGSH}(\mathrm{RR}=1.57, p=0.19)$.

\section{Discussion}

Typically, in case-control studies, healthy volunteers are included in the control group, but in this study we compared acute stroke with cerebral microangiopathy. This is due to the following consideration: acute cerebral ischemia itself is a factor causing rapid and significant changes in thiol homeostasis not only in the brain, but also in the blood plasma [13, 14]. Therefore, to more clearly reflect the effect of IS on plasma GSH homeostasis, it was more appropriate to use as a comparison group not healthy people characterized by a "normal" balance of thiols, but people at risk of stroke, which include patients with cerebral microangiopathy.

According to a number of studies, normal levels of tGSH and rGSH in plasma are $~ 4.3-9.1$ and $\sim 1.5-4.3 \mu \mathrm{M}$ [24-26]. Our study has shown that in patients with acute stroke, these indicators are significantly lower than the normal level, and they are also lower than in patients with cerebral microangiopathy. This is consistent with the results of experimental studies, which showed that acute cerebral ischemia is accompanied by a significant decrease in the values of GSH [27]. This is due to a decrease in its synthesis and / or intensive consumption by reason of a significant increase in the activity of GSHdependent enzymes, for example, glutathione peroxidase [28]. In addition, it was found that the activity of this enzyme in erythrocytes significantly decreased the risk of stroke [29]. In addition, the level of glutathione peroxidase was negatively correlated with NIHSS on admission and after 7 days from stroke [17]. Polymorphism of another GSH-metabolizing enzyme, glutathione S-transferase, is also associated with the risk of stroke [30].

It was shown on the rat brain ischemia/reperfusion model that intensification of GSH synthesis or its consumption attenuated oxidative stress and protected against the exacerbated brain damage [31, 32]. Part of GSH reacts with NO to form unstable nitrosoglutathione (GSNO). Pre-clinical studies showed positive neuroprotective effect of GSNO in animal stroke models [33]. Other works have shown that glutathione-ethyl ester is able to effectively prevent oxidative damage of mitochondrial complex I caused by ischemia/reperfusion and thereby reduces the infarct size and improved neurological outcome [34, 35]. However, research on the effectiveness of GSH and the significance of its level for the diagnosis of various subtypes of stroke has not yet been conducted.

The results of our study showed that significant differences in the content of total aminothiols (cysteine, homocysteine, and GSH) exist between LA and CE groups. LA was characterized by elevated plasma levels of these metabolites, but a detailed analysis of these results is beyond the scope of this article. A lot of studies on the relationship between plasma total homocysteine level and stroke subtypes have been published; however, the results have been varied. Homocysteine has been recognized as an independent risk factor for stroke, and many studies have found a high incidence of elevated levels (hyperhomocysteinemia-HHcy) in all stroke subtypes [36-39]. An early study found no difference in this indicator among the subtypes of stroke [36]. However, later, larger work and meta-analyses showed that the incidence of HHcy is higher in LA than in other subtypes, including CE [37, 38, 40-42], which is consistent with our results. In addition, $\mathrm{CE}$ is generally not characterized by a high incidence of HHcy [40], and an inverse association of CE risk with total homocysteine levels was revealed [43]. The high homocysteine level in LA most likely reflects its proatherogenic role in the pathogenesis of stroke, and may also explain the increased levels of cysteine and GSH in plasma, since a significant part of homocysteine is converted to cysteine, and the latter, in turn, is required for the formation of GSH. In contrast, it was reported that the homocysteine level was higher in CE than in LA [39]. There is a lack of data regarding the association of tGSH levels in different subtypes of stroke, which is due to the low content of this metabolite and the lack of sensitive routine methods for its determination in blood plasma. It has only recently been shown that low GSH levels are associated with neurological deficits in acute ischemic stroke [44].

Our results showed that low t- and rGSH levels may be a risk factor for increased neurological deficits in patients with LA, but not with CE stroke, even though CE was more commonly characterized by low tGSH level. In addition, a low level of tGSH is obviously associated with an increased risk of poor functional outcome in short term in LA, but in the case of CE this factor may play, on the contrary, a positive role.

In addition, a low level of rGSH can be considered a risk marker for decreased independence in patients with LA, but not CE in short term. It is unlikely that these results could be due to age, sex differences, or 
the different incidence of chronic cerebral ischemia between these groups of patients. It is highly likely that cysteine and/or homocysteine have a significant effect on the association of low GSH with stroke severity, but the limited sample size of this study did not allow such an analysis.

Correction of GSH levels, especially in LA, can have a positive therapeutic effect. Recently, in a small study, the positive effect of taking $\mathrm{N}$-acetylcysteine, a compound that intensifies GSH synthesis, was shown on functional status and neurological deficits 3 months after acute ischemic stroke [45]. In addition, it was shown that the infusion of $\mathrm{N}$-acetylcysteine led to a rapid rise in GSH levels in the brain [46]. However, it is not yet clear whether its protective effect was caused by the synthesis of GSH or the drug's own antioxidant activity. The authors of these works also did not analyze its effectiveness among patients with LA and CE separately.

An increase in the level of total homocysteine and cysteine in LA patients may indicate a decrease in the bioavailability of cysteine as a rate-limiting substrate for GSH synthesis due to inhibition of the transport and/ or synthesis of cysteine in cells. It is also interesting to note that in the LA group, in contrast to the CE group, we did not find significant associations of the total level of glutathione with cysteine and homocysteine, which may also indicate that this subtype of stroke is more commonly associated with systemic disruption of aminothiols metabolism.

The present work, according to our data, being the first pilot study to identify the diagnostic and prognostic potential of GSH in the subtypes of ischemic stroke, was carried out on a rather limited number of subjects, which did not allow us to investigate additional factors affecting the association of GSH with clinical indicators of stroke. Additional studies are required to help identify in more detail the role of plasma GSH as a risk marker for the unfavorable course of atherothrombotic stroke or explain its involvement in the pathology of stroke.

\section{Conclusions}

In our study, significant differences were found in the composition of blood plasma aminothiols between LA and $C E$ group. Although both of these stroke subtypes were characterized by decreased levels of tGSH and rGSH in plasma, low levels of these analytes were promising markers of risk for increased neurological deficits and decreased functional independence only in patients with LA. In contrast, a low tGSH level can be considered a marker of a favorable short-term prognosis for cardioembolic stroke.

\section{Abbreviations}

AOR: Adjusted odds ratio; BI: Bartel index; CE: Cardioembolic stroke; Cl: 95\% Coincidence interval; GSH: Glutathione; GSNO: Nitrosoglutathione; HHcy: Hyperhomocysteinemia; LA: Large-artery atherosclerosis; MR: Magnetic resonance; MRI: Magnetic resonance imaging; mRs: Modified Rankin scale; NIHSS: National Institutes of Health Stroke Scale; OR: Unadjusted odds ratio; rGSH: Reduced glutathione; RR: Risk relative risk; tGSH: Total glutathione; TOAST:Trial of ORG 10172 in acute stroke treatment.

\section{Acknowledgements \\ Not applicable.}

\section{Authors' contributions}

Research idea - MAP and AAK, study design and data collection-MYM, FRO and ETS, data analysis and interpretation-AVI, KAN, VVA, manuscript writing-AVI, MYM, MPK, general editing and final revision-AVI. All authors read and approved the final manuscript.

\section{Funding}

No funds, Grants, or other support was received.

\section{Availability of data and materials}

The data sets used and/or analysed during the current study are available from the corresponding author on reasonable request.

\section{Declarations}

\section{Ethics approval and consent to participate}

Study approval was from the Ethics Committee of the Research Center of Neurology (Moscow, Russia). An oral and written consent was obtained from the patients who participated in this research.

\section{Consent for publication}

All participants approved the submission and publication.

\section{Competing interests}

The authors declare that they have no competing interests.

\section{Author details}

'Department of Molecular and Cellular Pathophysiology, Institute of General Pathology and Pathophysiology, Baltiyskaya st., 8, 125315 Moscow, Russia. ${ }^{2}$ Research Center of Neurology, Moscow, Russia. ${ }^{3}$ Moscow State Medical and Dental University, Moscow, Russia. ${ }^{4}$ Sechenov First Moscow State Medical University (Sechenov University), Moscow, Russia. ${ }^{5}$ Russian Medical Academy of Postdoctoral Education, 125993 Moscow, Russia.

Received: 11 August 2021 Accepted: 14 January 2022

Published online: 02 February 2022

References

1. Kolominsky-Rabas PL, Weber M, Gefeller O, et al. Epidemiology of ischemic stroke subtypes according to TOAST criteria: incidence, recurrence, and long-term survival in ischemic stroke subtypes: a populationbased study. Stroke. 2001;32:2735-40. https://doi.org/10.1161/hs1201. 100209.

2. Martinez-Revelles S, Jiménez-Altayó F, Caracuel L, et al. Endothelial dysfunction in rat mesenteric resistance artery after transient middle cerebral artery occlusion. J Pharmacol Exp Ther. 2008;325:363-9. https:// doi.org/10.1124/jpet.107.134619.

3. Allen $\mathrm{CL}$, Bayraktutan U. Oxidative stress and its role in the pathogenesis of ischaemic stroke. Int J Stroke. 2009;4(6):461-70. https://doi.org/10. 1111/j.1747-4949.2009.00387.x.

4. Cherubini A, Polidori MC, Bregnocchi M, et al. Antioxidant profile and early outcome in stroke patients. Stroke. 2000;31:2295-300. https://doi. org/10.1161/01.str.31.10.2295

5. Milanlioglu A, Aslan M, Ozkol H, et al. Serum antioxidant enzymes activities and oxidative stress levels in patients with acute ischemic stroke: 
influence on neurological status and outcome. Wien Klin Wochenschr. 2016;128:169-74. https://doi.org/10.1007/s00508-015-0742-6.

6. Liu Z, Zhu Z, Zhao J, et al. Malondialdehyde: a novel predictive biomarker for post-stroke depression. J Affect Disord. 2017;220:95-101. https://doi. org/10.1016/j.jad.2017.05.023.

7. Yeh TT, Hsieh YW, Wu CY, et al. A preliminary investigation of the association of sleep with inflammation and oxidative stress biomarkers and functional outcomes after stroke rehabilitation. Sci Rep. 2017;7:8634. https://doi.org/10.1038/s41598-017-08931-w.

8. Ghonimi NAM, Mahdy ME, Abdel Salam OA. Total antioxidant capacity predicts outcome in acute ischemic stroke subtypes in Egyptian patients. J Stroke Cerebrovasc Dis. 2019;28(7):1911-7. https://doi.org/10.1016/j. jstrokecerebrovasdis.2019.03.053.

9. Ishibashi N, Prokopenko O, Reuhl KR, Mirochnitchenko O. Inflammatory response and glutathione peroxidase in a model of stroke. J Immunol. 2002;168:1926-33. https://doi.org/10.4049/jimmunol.168.4.1926.

10. Paterson $P G$, Juurlink $B H$. Nutritional regulation of glutathione in stroke. Neurotox Res. 1999;1:99-112. https://doi.org/10.1007/BF03033274.

11. Sedlak TW, Paul BD, Parker GM, et al. The glutathione cycle shapes synaptic glutamate activity. Proc Natl Acad Sci U S A. 2019;116:2701-6. https:// doi.org/10.1073/pnas.1817885116

12. Michelet F, Gueguen R, Leroy $P$, et al. Blood and plasma glutathione measured in healthy subjects by HPLC: relation to sex, aging, biological variables, and life habits. Clin Chem. 1995;41:1509-17.

13. Maksimova MY, Ivanov AV, Virus ED, et al. Disturbance of thiol/disulfide aminothiols homeostasis in patients with acute ischemic stroke stroke: preliminary findings. Clin Neurol Neurosurg. 2019;183: 105393. https:// doi.org/10.1016/j.clineuro.2019.105393.

14. Ivanov AV, Alexandrin WV, Paltsyn AA, et al. Plasma low-molecular-weight thiol/disulphide homeostasis as an early indicator of global and focal cerebral ischaemia. Redox Rep. 2017:22:460-6. https://doi.org/10.1080/ 13510002.2017.1311464.

15. Ivanov AV, Alexandrin W, Paltsyn AA, et al. Metoprolol and nebivolol prevent the decline of the redox status of low-molecular-weight aminothiols in blood plasma of rats during acute cerebral ischemia. J Cardiovasc Pharmacol. 2018;72:195-203. https://doi.org/10.1097/FJC.0000000000 000616.

16. An L, Dani KA, Shen J, Warach S. Natural History of Stroke Investigators. Pilot results of in vivo brain glutathione measurements in stroke patients. J Cereb Blood Flow Metab. 2012;32:2118-21. https://doi.org/10.1038/ jcbfm.2012.127.

17. Zimmermann C, Winnefeld K, Streck S, et al. Antioxidant status in acute stroke patients and patients at stroke risk. Eur Neurol. 2004;51:157-61. https://doi.org/10.1159/000077662.

18. Ozkul A, Akyol A, Yenisey C, et al. Oxidative stress in acute ischemic stroke. J Clin Neurosci. 2007;14:1062-6. https://doi.org/10.1016/j.jocn.2006.11. 008.

19. Gerreth P, Maciejczyk M, Zalewska A, et al. Comprehensive evaluation of the oral health status, salivary gland function, and oxidative stress in the saliva of patients with subacute phase of stroke: a case-control study. J Clin Med. 2020;9:2252. https://doi.org/10.3390/jcm9072252.

20. Adams HP Jr, Bendixen BH, Kappelle LJ, et al. Classification of subtype of acute ischemic stroke. Definitions for use in a multicenter clinical trial. TOAST. Trial of Org 10172 in Acute Stroke Treatment. Stroke. 1993;24:3541. https://doi.org/10.1161/01.str.24.1.35 (PMID: 7678184).

21. Brott T, Adams HP Jr, Olinger CP, et al. Measurements of acute cerebral infarction: a clinical examination scale. Stroke. 1989:20:864-70. https:// doi.org/10.1161/01.str.20.7.864.

22. Sulter G, Steen C, De Keyser J. Use of the Barthel index and modified Rankin scale in acute stroke trials. Stroke. 1999;30:1538-41. https://doi. org/10.1161/01.str.30.8.1538.

23. Mahoney Fl, Barthel DW. Functional evaluation: the Barthel index. Md State Med J. 1965;14:61-5.

24. Svardal AM, Mansoor MA, Ueland PM. Determination of reduced, oxidized, and protein-bound glutathione in human plasma with precolumn derivatization with monobromobimane and liquid chromatography. Anal Biochem. 1990;184:338-46. https://doi.org/10.1016/0003-2697(90) 90691-2.

25. Andersson A, Isaksson A, Brattström L, Hultberg B. Homocysteine and other thiols determined in plasma by HPLC and thiol-specific postcolumn derivatization. Clin Chem. 1993:39:1590-7.
26. Williams RH, Maggiore JA, Reynolds RD, Helgason CM. Novel approach for the determination of the redox status of homocysteine and other aminothiols in plasma from healthy subjects and patients with ischemic stroke. Clin Chem. 2001;47:1031-9.

27. Raza SS, Khan MM, Ahmad A, et al. Hesperidin ameliorates functional and histological outcome and reduces neuroinflammation in experimental stroke. Brain Res. 2011;1420:93-105. https://doi.org/10.1016/j.brainres. 2011.08.047.

28. Žitňanová I, Šiarnik P, Kollár B, et al. Oxidative stress markers and their dynamic changes in patients after acute ischemic stroke. Oxid Med Cell Longev. 2016;2016:9761697. https://doi.org/10.1155/2016/9761697.

29. Chehaibi K, Trabelsi I, Mahdouani K, Slimane MN. Correlation of oxidative stress parameters and inflammatory markers in ischemic stroke patients. J Stroke Cerebrovasc Dis. 2016;25:2585-93. https://doi.org/10.1016/j.jstro kecerebrovasdis.2016.06.042.

30. Wang R, Wang Y, Wang J, Yang K. Association of glutathione S-transferase $\mathrm{T} 1$ and $\mathrm{M} 1$ gene polymorphisms with ischemic stroke risk in the Chinese Han population. Neural Regen Res. 2012;7:1420-7. https://doi.org/10. 3969/j.issn. 1673-5374.2012.18.009.

31. Song J, Park J, Oh Y, Lee JE. Glutathione suppresses cerebral infarct volume and cell death after ischemic injury: involvement of $\mathrm{FOXO} 3$ inactivation and BCl2 expression. Oxid Med Cell Longev. 2015;2015: 426069. https://doi.org/10.1155/2015/426069.

32. Wang B, Aw TY, Stokes KY. The protection conferred against ischemia-reperfusion injury in the diabetic brain by $\mathrm{N}$-acetylcysteine is associated with decreased dicarbonyl stress. Free Radic Biol Med. 2016;96:89-98. https:// doi.org/10.1016/j.freeradbiomed.2016.03.038.

33. Liu S, Zheng H, Yu W, et al. Investigation of S-Nitrosoglutathione in stroke: a systematic review and meta-analysis of literature in pre-clinical and clinical research. Exp Neurol. 2020;328: 113262. https://doi.org/10.1016/j. expneurol.2020.113262.

34. Anderson MF, Nilsson M, Eriksson PS, Sims NR. Glutathione monoethyl ester provides neuroprotection in a rat model of stroke. Neurosci Lett. 2004;354(2):163-5. https://doi.org/10.1016/j.neulet.2003.09.067.

35. Kahl A, Stepanova A, Konrad C, et al. Critical role of flavin and glutathione in complex I-mediated bioenergetic failure in brain ischemia/reperfusion injury. Stroke. 2018;49:1223-31. https://doi.org/10.1161/STROKEAHA.117. 019687.

36. Brattström L, Lindgren A, Israelsson B, et al. Hyperhomocysteinaemia in stroke: prevalence, cause, and relationships to type of stroke and stroke risk factors. Eur J Clin Invest. 1992;22:214-21. https://doi.org/10.1111/j. 1365-2362.1992.tb01829x.

37. Zhang $T$, Jiang $Y$, Zhang $S$, et al. The association between homocysteine and ischemic stroke subtypes in Chinese: a meta-analysis. Medicine (Baltimore). 2020;99: e19467. https://doi.org/10.1097/MD.0000000000019467.

38. Parnetti L, Caso V, Santucci A, et al. Mild hyperhomocysteinemia is a risk-factor in all etiological subtypes of stroke. Neurol Sci. 2004;25:13-7. https://doi.org/10.1007/s10072-004-0219-5.

39. Ashjazadeh N, Fathi M, Shariat A. Evaluation of homocysteine level as a risk factor among patients with ischemic stroke and its subtypes. Iran J Med Sci. 2013;38:233-9.

40. Eikelboom JW, Hankey GJ, Anand SS, et al. Association between high homocyst(e)ine and ischemic stroke due to large- and small-artery disease but not other etiologic subtypes of ischemic stroke. Stroke. 2000;31:1069-75. https://doi.org/10.1161/01.str.31.5.1069.

41. Gungor L, Polat M, Ozberk MB, et al. Which ischemic stroke subtype is associated with hyperhomocysteinemia? J Stroke Cerebrovasc Dis. 2018;27:1921-9. https://doi.org/10.1016/j.jstrokecerebrovasdis.2018.02. 033.

42. Ahmed S, Bogiatzi C, Hackam DG, et al. Vitamin B 12 deficiency and hyperhomocysteinaemia in outpatients with stroke or transient ischaemic attack: a cohort study at an academic medical centre. BMJ Open. 2019;9: e026564. https://doi.org/10.1136/bmjopen-2018-026564.

43. Bushnell CD, Goldstein LB. Homocysteine testing in patients with acute ischemic stroke. Neurology. 2002;59:1541-6. https://doi.org/10.1212/01. wnl.0000034173.19459.d0.

44. Maksimova MY, Ivanov AV, Virus ED, et al. Impact of glutathione on acute ischemic stroke severity and outcome: possible role of aminothiols redox status. Redox Rep. 2021;26:117-23. https://doi.org/10.1080/13510002. 2021.1952819. 
45. Sabetghadam M, Mazdeh M, Abolfathi P, et al. Evidence for a beneficial effect of oral $\mathrm{N}$; acetylcysteine on functional outcomes and inflammatory biomarkers in patients with acute ischemic stroke. Neuropsychiatr Dis Treat. 2020;16(1265):1278. https://doi.org/10.2147/NDT.S241497.

46. Moss HG, Brown TR, Wiest DB, Jenkins DD. N-Acetylcysteine rapidly replenishes central nervous system glutathione measured via magnetic resonance spectroscopy in human neonates with hypoxic-ischemic encephalopathy. J Cereb Blood Flow Metab. 2018;38:950-8. https://doi. org/10.1177/0271678X18765828.

\section{Publisher's Note}

Springer Nature remains neutral with regard to jurisdictional claims in published maps and institutional affiliations.

\section{Submit your manuscript to a SpringerOpen ${ }^{\circ}$ journal and benefit from:}

- Convenient online submission

- Rigorous peer review

- Open access: articles freely available online

- High visibility within the field

- Retaining the copyright to your article

Submit your next manuscript at $\boldsymbol{\nabla}$ springeropen.com 